\title{
Simplified Deformation Analysis of 3D-body by First Order Analysis*
}

\author{
Md. Shawkut ALI KHAN ${ }^{* *}$, Koetsu YAMAZAKI** ${ }^{* * * * *}$ Kosuke ODA ${ }^{* * *}$ \\ and Kazuhiko HIROOKA ${ }^{* * *}$ \\ ** Graduate School of Natural Science and Technology, Kanazawa University \\ Kakuma-machi, Kanazawa, 920-1192, Japan \\ E-mail : shawkut19@gmail.com, yamazaki@t.kanazawa-u.ac.jp \\ ${ }^{* * *}$ Mitsubishi Electric Co. Ltd., Hamayama-dori, Hyogo-ku, Kobe, 652-0871, Japan \\ ${ }^{* * * *}$ Aisin Seiki Co. Ltd., 2-1, Asahi-machi, Kariya, Aichi, 448-8650, Japan
}

\begin{abstract}
This paper proposes a simplified analysis model of complex solid structure for three-dimensional bodies (3D-bodies) in the First Order Analysis (FOA). A simplified finite element composed of the beam elements is suggested for the deformation analysis of 3D-bodies by extending concept of conforming beam element for the finite element method (FEM). When a complex solid structure is simplified to an assemblage of simple elements applied element forces such as axial forces, shear forces, bending moments as well as twisting moments, the total stiffness equation for the FOA of the solid structure is constructed approximately and solved for the given boundary and loading conditions by the usual manner of the FEM. Then, the total deformation of the complex structure is estimated by the simplified model and the element forces are calculated in the element level. It is found that the finite element analysis via developed simplified element analysis can estimate deformations of complex 3D-bodies in a rational precision, and the results of the analysis are useful for considering the load transmitting path of the structure and for designing a better structure by changing the stiffness of structural components.
\end{abstract}

Key words: First Order Analysis, Finite Element Analysis, Design Engineering.

\section{Introduction}

Computer-aided engineering (CAE) by Lemon et al. ${ }^{(1)}$ has been widely accepted by the industrial designers and the simulation engineers. Many efforts to assure the analysis precision and convergence according to the mesh fineness have been done by the research to develop better bending elements by introducing the mixed approach and non-conforming formulation ${ }^{(2),(3)}$. Finally, it has been found that the conforming beam element which assures the continuity of the first derivatives of deflection, and isoparametric shell elements which adopt a kind of reduced numerical integration have resolved these issues. Moreover the structural optimization software such as GENESIS is frequently used for the design optimization that satisfies the design specification by modeling the detailed structural elements.

However, it is difficult for the designers to extract some knowledge why the final design obtained by the optimization software is optimum, therefore no design knowledge is accumulated in the designer's brain as skillful engineering know-how. To overcome this issue, a new concept of design tool so called the First Order Analysis (FOA) has been proposed by Nishigaki et al. ${ }^{(4),(5)}$ to treat automotive body structures by a low-fidelity model of skeletal structures which consists of beams and panels. The developed FOA tool 
provides a useful aid to design the load transmission paths and topology optimization of skeletal structures at upstream stage of design processes, because the tool includes a variety of graphical interfaces using MS Excel for the design engineers and powerful topology optimization module.

However, the FOA proposed by Nishigaki et al. ${ }^{(4)}{ }^{(5)}$ includes only the beam and panel elements as design elements, it is not enough to simplify the design model of complicate general solid structures such as machine tools in all kinds of structural design optimization. Moreover, the suggested FOA can treat only the rigidity of the body structure. On the other hand, some researches have also been tried to construct the low-fidelity CAE models to reduce the computational cost for the optimization of the complicate structural design of high-fidelity models ${ }^{(6),(7)}$. However, it is difficult to establish a unified theory and manner to construct low-fidelity models for the complicate 3D-bodies.

When we consider the optimum design of continuum body in the upstream design process, the stress concentration as well as the structural rigidity should be taken into account in the design process and the FOA may be extended to provide such ability for the designers. The final goal of a series of this research is to develop a new FOA concept, which can apply to the structural analysis and the design optimization of general solid structures considering the deformation/rigidity of the structure as well as the stress concentration. Then the main issues of FOA for the continuum 3D-bodies are:

(Issue 1) to predict the total deformation and the load transmission paths as well as the stress concentration of complicate structures by the simplified model of low-fidelity, and

(Issue 2) to assist the design engineers to optimize the structure, to make them understandable how the loads are transmitted rationally to the supports, and which parameters are important for the structural optimization.

In the practical cases, the design engineers are expected to design and to produce creative design products, and they have to check the possibility of many kinds of structural candidates of very complicate machine elements. To realize this design process and to assist them for the creative and interactive thinking on the computer, a software embedded the mentioned steps above with graphical interface is expected to develop. To achieve such kinds of design environment for the engineers, it is required;

(Requirement 1) to offer functions (user interface) to define the simplified models from the complex real three-dimensional objects, and

(Requirement 2) to calculate the equivalent stiffness for the given design parameters.

It is also desired for the assistance of designing processes;

(Requirement 3) to prepare the element stiffness library and stress concentration factor library as functions of the design parameters, and the simplified element forces for many kinds of element shapes and variable cross sections to predict some change of stress concentration factors.

The purpose of this paper is to develop a simplified deformation analysis methodology and its element for the 3D-body structures in the FOA. At first, a simplified finite element that can express tensile, compressive, torsion and bending deformations of the body by extending simple concept of conforming beam element is proposed. Then, the proposed FOA method is applied to a couple of basic analysis examples, and the numerical results are compared with the elementary theory to discuss the precision and estimation ability. Then, the proposed FOA method is applied to estimate approximate deformations of complicate 3D-bodies, and the analyzed results are compared with the usual FEM(finite element method) results. From the examination of the effectiveness of the proposed element, it is found the proposed FOA method is useful for considering the load transmitting path of the structure and for designing a better structure by changing the stiffness of structural components. 


\section{Formulation of Simplified 3D-FOA Element}

\subsection{FOA of Three-dimensional Body}

The main themes of 3D-body FOA are: (a) to supply analysis methodology of low-fidelity model of complicate 3D-bodies, (b) to prepare some graphic interfaces for designers using such as Microsoft/Excel ${ }^{(4),(5),(8)}$ for the design assistance and optimization, and (c) to offer interactive design function to show the effects such as the design parameter changes and load transmitting paths. Figure 1 shows an image of 3D-body FOA by comparing with the fine CAE model.

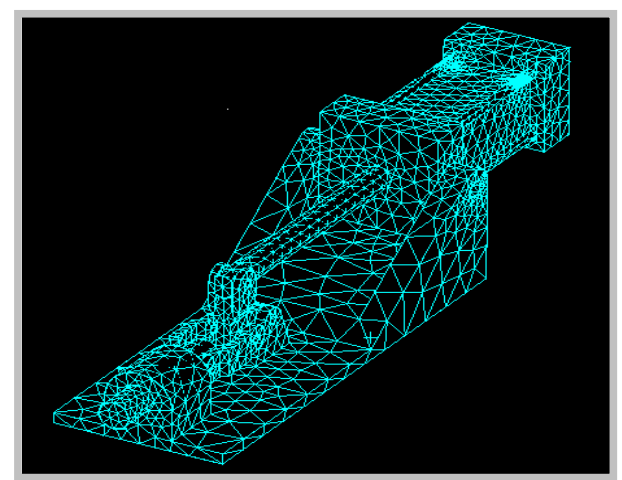

(a) Finite element mesh for $\mathrm{CAE}$

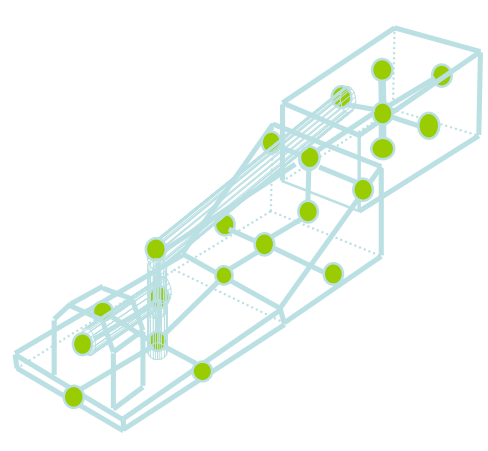

(b) Descritization for FOA

Fig. 1 Comparison of FOA model and FEM model

\subsection{Construction of FOA Model for Continuum Body}

Let us start discussions of the FOA model construction for the planar continuum body because of simplicity. For a complicate continuum it is difficult to understand directly how the load is transmitted to the supports, and the influence of design variable changes to the total deformation or the stress concentration factor. So, the FOA for the continuum body as shown in Fig.1(b) is constructed to satisfy these requirements by the following steps:

(Procedure 1) Simplify the whole complex model to an assemblage of a various kinds of simple conforming elements. Figure 2(a) shows the total model, and Fig.2(b) illustrates the simplified one as the assemblage of simple elements with wholes, notches and step changes of cross sections.

(Procedure 2) Decompose assembled elements to a set of simple elements applied forces such as axial and shear forces, bending and twisting moments. If the relationship between the element stiffness and the design parameters is prepared for a various kinds of simplified elements in the computer library, we may invoke the stiffness matrix for the specified design parameters.

(Procedure 3) Construct the total stiffness equation as an assemblage of the simple elements. For example, if a planar beam element with a V-notch has several design parameters such as width $W$, a half of the distance $a$ between the two notch roots, root radius of notch $\rho$, and notch angle $\alpha$ as shown in Fig.2(c), the element stiffness equation is given such as

$$
\mathrm{F}_{\mathrm{e}}=\mathrm{K}_{\mathrm{e}} \mathrm{U}_{\mathrm{e}}
$$

where $\mathbf{F}_{\mathrm{e}}=\left(P_{1}, V_{1}, M_{1}, P_{2}, V_{2}, M_{2}\right)^{\mathrm{T}}, \mathbf{U}_{\mathrm{e}}=\left(u_{1}, v_{1}, \theta_{1}, u_{2}, v_{2}, \theta_{2}\right)^{\mathrm{T}}$ when $P_{i}, V_{i}, M_{i}(i=1,2)$ denote the axial forces, the shear forces and the bending moments, and $u_{i}, v_{i}, \theta_{i}(i=1,2)$ denote the axial and the transverse displacements, and the rotations of both ends, respectively. The stiffness matrix of simplified element should be prepared as a function of the design parameters as $\mathbf{K}_{\mathrm{e}}=\mathbf{K}_{\mathrm{e}}(\rho, a, W, \alpha)$ in advance. Then, the element stiffness matrix is assembled to obtain the total stiffness matrix, and thereby solved for the total 
displacement of the structure under the given loading and boundary conditions as usual manner of the finite element analysis. Then, the structural deformation of whole structure is predicted roughly.

(Procedure 4) Calculate the element forces to predict the stress concentration for the given design parameters. To do so, some sophisticated formulas are prepared for the maximum stress for the typical element including design parameters.

(Procedure 5) Display the deformation and element forces. Then, the designer can observe how the load is transmitted, and also how much the stress concentrates at each element.

(Procedure 6) Change the structural topology if needed and optimize the design parameters. When we have to change any dimension or shape, we may go to the Procedure 2 or to the Procedure 1 if required the structural model change.
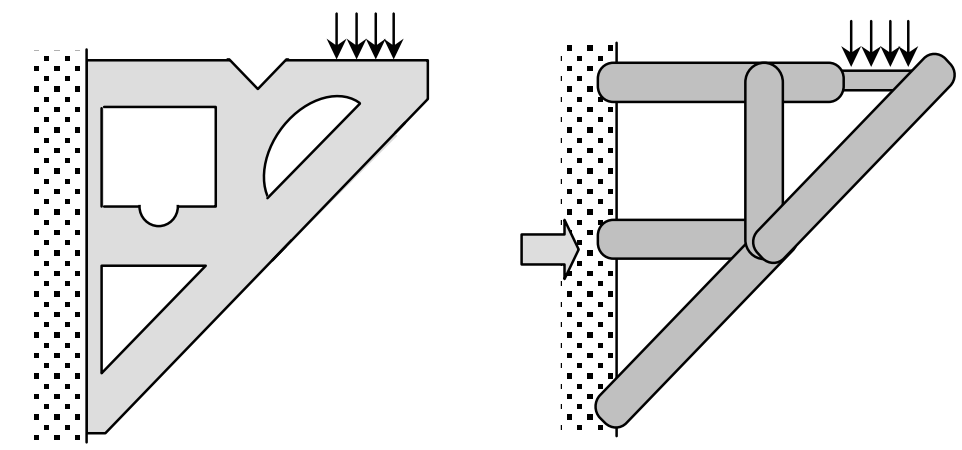

(a) Real structure

(b) Simplified structure
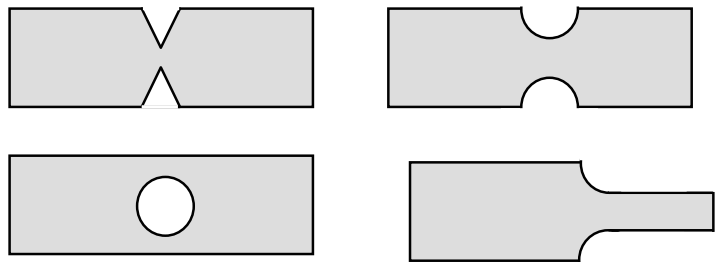

(c) Element library

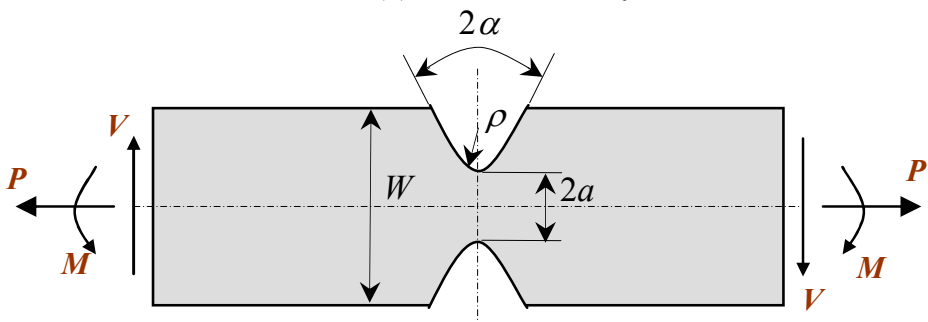

(d) An element with notches applied forces

Fig.2 Decomposition of planar continuum body

\subsection{Derivation of Simplified Finite Element Stiffness}

When we want to obtain precise stiffness of 3D-body, the analysis model has to be descritized into fine meshes by the usual finite elements. On the other hand, the continuum body modeled by an equivalent beam element that transmits only tension/compression, torsion and bending is enough to estimate roughly the deformation of complicate 3D-bodies and load transmitting path. To develop such kind of simplified finite element, it is assumed that an approximate deformation mode of complex continuum body is expressed by the combination of tensile, compressive, bending and torsional basic deformations. As the simplest approximation example of 3D-body deformation, suppose that a general hexahedral block shown in Fig.3(a) is in a state of uniform deformation. The hexahedral block consists of seven representative nodes, six nodes of which are located on the center point of hexahedral edge surfaces, and the $7^{\text {th }}$ node of which is located at the center of hexahedron. The distances $L_{x}, L_{y}$ and $L_{z}$ in Fig.3(b) denote lengths between node 1 and node 
2, nodes 3,5 and nodes 4,6. Each node has 6 degrees of freedom of displacement $\left(u_{i}, v_{i}\right.$, $\left.w_{i}, \theta_{x i}, \theta_{y i}, \theta_{z i}\right)(i=1,2,3 \ldots, 7)$ in the local coordinate systems $\left(x^{\prime}, y^{\prime}, z^{\prime}\right)$ of an element. Corresponding to the displacement components, axial forces $\left(Q_{x i}, Q_{y i}, Q_{z i}\right)$, and moments $\left(T_{x i}, T_{y i}, T_{z i}\right)$ are considered at each node. When we regard a hexahedron as an assembled structure of six beams which consist of elements connecting the nodes at the center of hexahedral edge surfaces and a center node of hexahedron, the stiffness of hexahedron is derived by differentiating the total potential energy $\pi$ of the hexahedron with respect to each displacement component ${ }^{(9)(10)}$.

Let us consider the total potential energy $\pi$ of simple deformation such as tension/compression, bending and torsion under Bernoulli-Euler beam theory. When a beam consists of nodes 1 and 7 which take the deflection and the rotation as nodal unknown variables separately, the stiffness equation in the axial direction is derived by assuming the linear interpolating function as

$$
\frac{\partial \pi}{\partial u_{1}}=\frac{E A}{L_{x} / 2}\left(u_{1}-u_{7}\right)-Q_{x 1}=0
$$

By considering the potential energy for bending and assuming Hermite shape functions for the deflection to keep the conforming condition, we can derive stiffness equation as follows;

$$
\begin{aligned}
& \frac{\partial \pi}{\partial w_{1}}=\frac{12 E I_{y}}{\left(L_{x} / 2\right)^{3}}\left(w_{1}-w_{7}\right)+\frac{6 E I_{y}}{\left(L_{x} / 2\right)^{2}}\left(\theta_{y 1}+\theta_{y 7}\right)-Q_{z 1}=0 \\
& \frac{\partial \pi}{\partial \theta_{y 1}}=\frac{6 E I_{y}}{\left(L_{x} / 2\right)^{2}}\left(w_{1}-w_{7}\right)+\frac{2 E I_{y}}{\left(L_{x} / 2\right)}\left(2 \theta_{y 1}+\theta_{y 7}\right)-T_{y 1}=0
\end{aligned}
$$

and for the torsional stiffness equation,

$$
\frac{\partial \pi}{\partial \theta_{x 1}}=\frac{C}{\left(L_{x} / 2\right)}\left(\theta_{x 1}-\theta_{x 7}\right)-T_{x 1}=0
$$

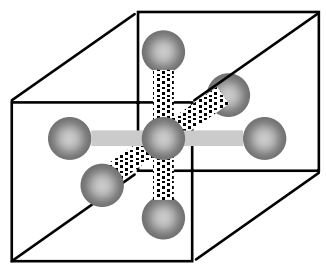

(a) Hexahedral block

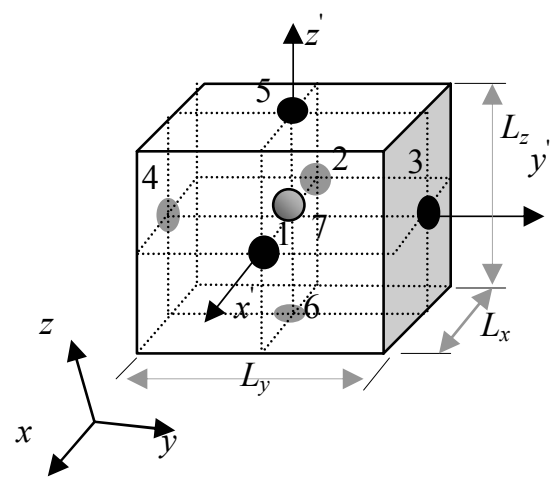

(b) Local coordinates and nodes

Fig.3 Simplified structural element

where, $E$ and $A$ are the Young's modulus and the cross-sectional area of the beam, $I_{y}$ is the second moment of inertia, and $C$ is the torsional rigidity. By the same way, the stiffness equations of other 5 beams are also derived. These stiffness equations are expressed by the equilibrium equation in the matrix form of Eq.(1), in which $\mathbf{K}_{\mathrm{e}}$ is the element stiffness matrix of 42 by 42 components for the simplified 3D-deformation analysis, where the proposed element stiffness does not take into account the influence of Poisson's ratio.

\subsection{Derivation of Equivalent Cross-sectional Area, $2^{\text {nd }}$ Moment of Inertia, Tortional Rigidity and Stiffness Matrix}


(a) Equivalent Cross-Sectional Area $\left(A_{e q}\right)$

When a load is applied on the end surface of regular quadrilateral block with uniform cross-section, the stiffness matrix can be calculated by the direct volume integration. However, for the non-uniform and irregular general shape as shown in Fig.4 we can not calculate the stiffness matrix directly by the usual formulas. Therefore, we have to consider more general shape by introducing coordinate transformation and numerical integration. Then, the equivalent stiffness matrix of general shape is derived by equating the strain energy of general solid model to the strain energy of simplified solid model with same volume.

Let us consider a block of general shape in Fig.4(a). When a load $P$ is applied on an element surface in the longitudinal direction, the strain energy for uniform shape element of beam which consists of nodes 1 and 7 can be express as follows:

$$
U_{e q}=\frac{E A_{e q-x}\left(u_{1}-u_{7}\right)^{2}}{2 L_{x}}
$$

where $A_{\text {eq- } x}, L_{x}$ and $\left(u_{1}-u_{7}\right)$ are the equivalent cross-sectional area, the length of the element in the $x$-direction and displacement, respectively. On the other hand, in the general case the strain energy can be expressed as follows:

$$
U_{\text {general }}=\frac{E\left(u_{1}-u_{7}\right)^{2}}{2 L_{x}^{2}} \int_{0}^{L} A(x) d x
$$

In the practical evaluation, by introducing local coordinates, $-1 \leq \xi, \eta, \zeta \leq 1$ we can get a general form of integration as

$$
I_{m n}(x(y, z))=\left.\int_{-1}^{1} \int_{-1}^{1} y^{m} z^{n}|J| d \eta d \zeta\right|_{a t x} \quad(m, n=0,1,2)
$$

where

$$
|J|=\left|\begin{array}{ll}
\frac{d y}{d \eta} & \frac{d z}{d \eta} \\
\frac{d y}{d \zeta} & \frac{d z}{d \zeta}
\end{array}\right|=\left(\frac{d y}{d \eta} \cdot \frac{d z}{d \zeta}-\frac{d z}{d \eta} \cdot \frac{d y}{d \zeta}\right)
$$

For example, an equivalent cross-section is given by $I_{00}$ when $m=n=0$, and equivalent second moment of inertia around $y$ - and $z$-axis are given by $I_{02}$ and $I_{20}$ when $m=0, n=2$ and $m=2, n=0$. By using Gauss quadrature,

$$
I_{m n}\left(x_{i}(y, z)\right)=\sum_{i=1}^{s m p} \sum_{j=1}^{s m p} w_{i} w_{j} y^{m}\left(\eta_{i}, \zeta_{j}\right) z^{n}\left(\eta_{i}, \zeta_{j}\right)\left|J\left(\eta_{i}, \zeta_{j}\right)\right|_{a t x=x_{i}}
$$

where $\operatorname{smp}$ and $w_{i}, w_{j}$ denote the integration order and corresponding weights in each direction. Then, the equation of strain energy $U_{\text {general }}$ becomes as follows:

$$
\begin{aligned}
& U_{\text {general }}=\left(\frac{L_{x}}{2}\right) \frac{E\left(u_{1}-u_{7}\right)^{2}}{2 L_{x}^{2}} \int_{-1}^{1} A(\xi) d \xi \\
& \frac{E\left(u_{1}-u_{7}\right)^{2}}{2 L_{x}^{2}}\left(\frac{L_{x}}{2}\right)_{i=1}^{s m p} w_{i} A\left(\xi_{i}\right)=\frac{E\left(u_{1}-u_{7}\right)^{2}}{2 L_{x}^{2}}\left(\frac{L_{x}}{2}\right) \sum_{i=1}^{s m p} w_{i} I_{00}\left(\xi_{i}\right)
\end{aligned}
$$

By equating $U_{e q}=U_{\text {general }}$, then we can get $A_{e q}$ as

$$
A_{e q-x}=\frac{1}{2} \sum_{i=1}^{s m p} w_{i} I_{00}\left(\xi_{i}\right)=\frac{1}{2} \sum_{i=1}^{s m p} w_{i}\left[\sum_{j=1}^{s m p} \sum_{k=1}^{s m p} w_{j} w_{k} y^{0}\left(\eta_{j}, \zeta_{k}\right) z^{0}\left(\eta_{j}, \zeta_{k}\right)\left|J\left(\eta_{j}, \zeta_{k}\right)\right|\right]
$$

(b) Equivalent Second Moment of Inertia $\left(I_{\mathrm{eq}}\right)$ 
Let us consider again a beam element which consists of two nodes. For the case of bending moment around the $y$-axis, when we assume cubic interpolating functions for the deflection by the terms of nodal defections and rotations around the axis as usual beam element, the strain energy for uniform shape is expressed as follows:

$$
U_{e q}=\frac{E I_{e q-y}}{2 L_{x}^{3}}\left(a^{2}+a b+\frac{b^{2}}{3}\right)
$$

where

$$
\begin{aligned}
& a=-6\left(w_{1}-w_{7}\right)-2 L_{x}\left(2 \theta_{y_{1}}+\theta_{y 7}\right) \\
& b=12\left(w_{1}-w_{7}\right)+6 L_{x}\left(\theta_{y_{1}}+\theta_{y 7}\right)
\end{aligned}
$$

On the other hand, for general shape the strain energy can be expressed by the following equations:

$$
U_{\text {general }}=\frac{E}{2 L_{x}^{3}}\left\{\left(\frac{a^{2}}{2}+\frac{a b}{2}+\frac{b^{2}}{8}\right) I_{e q-y}^{(0)}+\left(\frac{a b}{2}+\frac{b^{2}}{4}\right) I_{e q-y}^{(1)}+\frac{b^{2}}{8} I_{e q-y}^{(2)}\right\}
$$

where

$$
I_{e q-y}^{(0)}=\int_{-1}^{1} I_{20}(\xi) d \xi, \quad I_{e q-y}^{(1)}=\int_{-1}^{1} \xi I_{20}(\xi) d \xi, \quad I_{e q-y}^{(2)}=\int_{-1}^{1} \xi^{2} I_{20}(\xi) d \xi
$$

Unlike the calculation of $A_{e q-x}$, it is found that the equivalent second moment of inertia $I_{e q}$ can not be decided directly by equating Eq.(11) and Eq.(12).

(c) Tortional Rigidity:

Again for a rectangular element, whose cross-sectional area is given as $a \times b$ (where $a \geq b$ ), let us assume that the $2^{\text {nd }}$ moment of inertia equation by zero-order can be expressed as follows:

$$
I_{e q-y}=\frac{a b^{3}}{12}, \quad I_{e q-z}=\frac{a^{3} b}{12}
$$

Now solving Eq.(13) we can easily get the equivalent values of $a$ and $b$ and by substituting these values into Eq.(14), the equivalent torsional rigidity, $C_{e q}$. is obtained ${ }^{(10)}$ as follows:

$$
C_{e q-x}=\left\{\frac{1}{3}-0.21 \frac{b}{a}\left(1-\frac{b^{4}}{12 a^{4}}\right)\right\} a b^{3} G
$$

where $G$ denotes the shear modulus.

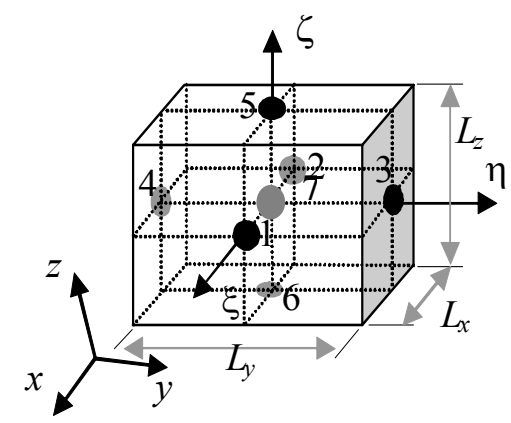

(a) General element

(b) Local coordinates

Fig.4 General structural element and its coordinate transformation

(d) Stiffness Matrix:

After calculating $A_{e q}, I_{e q}$, and $C_{e q}$, when we adopt a conforming element which takes the deflection and rotation of nodes the unknown variables separately, finally we can derive the total stiffness matrix as follows: 


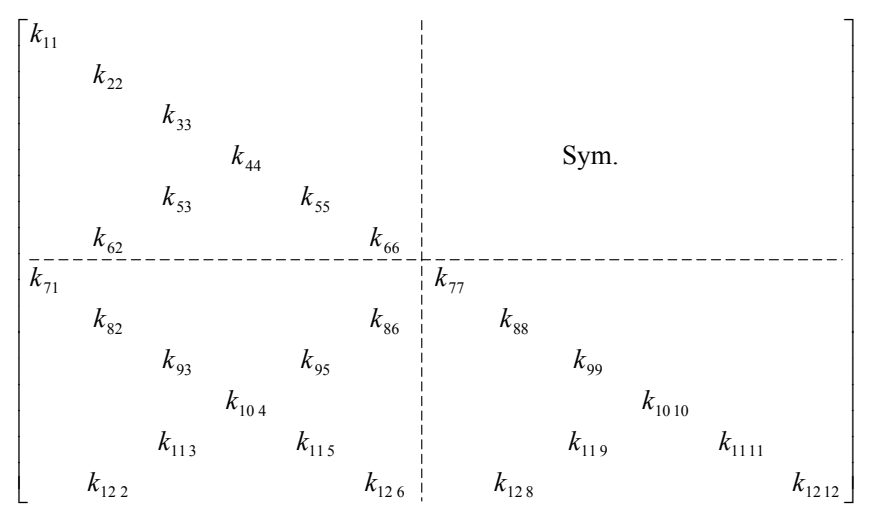

where

$$
\begin{aligned}
& k_{11}=\frac{E A_{e q-x}}{L_{x}}, \quad k_{22}=\frac{18 E I_{e q-z}^{(2)}}{L_{x}^{3}}, \quad k_{33}=\frac{18 E I_{e q-y}^{(2)}}{L_{x}^{3}}, \quad k_{44}=\frac{G C_{e q-x}}{L_{x}}, \quad k_{53}=\frac{E}{L_{x}^{2}}\left(3 I_{e q-y}^{(1)}-9 I_{e q-y}^{(2)}\right), \\
& k_{55}=\frac{E}{L_{x}}\left(\frac{1}{2} I_{e q-y}^{(0)}-3 I_{e q-y}^{(1)}+\frac{9}{2} I_{e q-y}^{(2)}\right), \quad k_{62}=\frac{E}{L_{x}^{2}}\left(-3 I_{e q-z}^{(1)}+9 I_{e q-z}^{(2)}\right), \\
& k_{66}=\frac{E}{L_{x}}\left(\frac{1}{2} I_{e q-z}^{(0)}-3 I_{e q-z}^{(1)}+\frac{9}{2} I_{e q-z}^{(2)}\right), \quad k_{71}=-\frac{E A_{e q-x}}{L_{x}}, \quad k_{77}=\frac{E A_{e q-x}}{L_{x}}, \quad k_{82}=-\frac{18 E I_{e q-z}^{(2)}}{L_{x}^{3}}, \\
& k_{86}=\frac{E}{L_{x}^{2}}\left(3 I_{e q-z}^{(1)}-9 I_{e q-z}^{(2)}\right), \quad k_{88}=\frac{18 E I_{e q-z}^{(2)}}{L_{x}^{3}}, \quad k_{93}=\frac{18 E I_{e q-y}^{(2)}}{L_{x}^{3}}, \quad k_{95}=\frac{E}{L_{x}{ }^{2}}\left(-3 I_{e q-y}^{(1)}+9 I_{e q-y}^{(2)}\right), \\
& k_{99}=\frac{18 E I_{e q-y}^{(2)}}{L_{x}^{3}}, \quad k_{104}=-\frac{G C_{e q-x}}{L_{x}}, \quad k_{1010}=\frac{G C_{e q-x}}{L_{x}}, \\
& k_{113}=\frac{E}{L_{x}{ }^{2}}\left(-3 I_{e q-y}^{(1)}-9 I_{e q-y}^{(2)}\right), \quad k_{115}=\frac{E}{L_{x}}\left(-\frac{1}{2} I_{e q-y}^{(0)}+\frac{9}{2} I_{e q-y}^{(2)}\right), \quad k_{119}=\frac{E}{L_{x}{ }^{2}}\left(-3 I_{e q-y}^{(1)}+9 I_{e q-y}^{(2)}\right), \\
& k_{1111}=\frac{E}{L_{x}}\left(\frac{1}{2} I_{e q-y}^{(0)}+3 I_{e q-y}^{(1)}+\frac{9}{2} I_{e q-y}^{(2)}\right), \quad k_{122}=\frac{E}{L_{x}^{2}}\left(3 I_{e q-z}^{(1)}+9 I_{e q-z}^{(2)}\right), \quad k_{128}=\frac{E}{L_{x}^{2}}\left(-3 I_{e q-z}^{(1)}-9 I_{e q-z}^{(2)}\right), \\
& k_{126}=\frac{E}{L_{x}}\left(-\frac{1}{2} I_{e q-z}^{(0)}+\frac{9}{2} I_{e q-z}^{(2)}\right), \quad k_{1212}=\frac{E}{L_{x}}\left(\frac{1}{2} I_{e q-z}^{(0)}-3 I_{e q-z}^{(1)}+\frac{9}{2} I_{e q-z}^{(2)}\right)
\end{aligned}
$$

By the same way, we can get the stiffness matrices for the other combination of nodes, such as the node 2 and node 7 , and etc. Then, the total stiffness matrix in the global coordinates system is obtained by the coordinate transformation from the local coordinates by the usual manner.

\section{Numerical Analysis by Proposed Structural Element Method}

The proposed 3D-FOA method described in the previous chapter has been applied to analyze several typical shaped examples. We have performed the simulation of deformation for a typical stepped beam, a tapered beam and an assembled structure applied several kinds of load such as tension/compression, bending/shear and torsion and their combination. The Young's modulus $E$ is assumed as $200 \mathrm{GPa}$ through the examples. The results obtained by the proposed method are compared with the results estimated by the elementary theory as well as the results obtained by the FEM. Then, the validity of proposed FOA method is discussed.

\subsection{Cantilever with Stepped Change of Cross-section}

First, we consider a cantilever structure with two stepped change of square cross-section as shown in Fig.5 $(L / h=5.0)$. Each step bar is descritized into one FOA element, and applied a tensile force $F$, a shear force $Q$ and a twisting moment $T$ at the tip, 
simultaneously. The numerical analysis results of tip elongation due to $F$, tip deflection due to $Q$, and twisting angle due to $T$ obtained by the proposed FOA are tabulated in Table 1 , when we put $h=4 \mathrm{~mm}, F=Q=1,000 \mathrm{~N}$ and $T=1,000 \mathrm{Nmm}$ as an example. These results are compared with those of elementary theory and the finite element analysis. In the finite element analysis, the stepped cantilever has been descritized into more than 110,000 elements and applied forces uniformly on the tip surface. Poisson's ratio is assumed as $v$ $=0.3$ for FEM. The FOA results agree very well with that of elementary theory. On the other

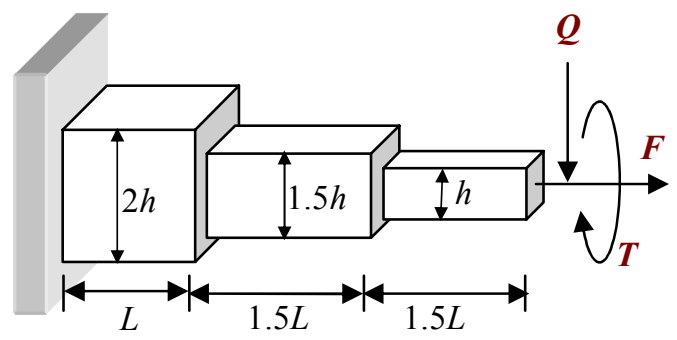

Fig. 5 Cantilever with stepped square cross-section applied tension, shear force and twisting moment on a tip

Table 1 Deformation analysis results of stepped beam comparing with those of the elementary theory and the finite element method.

\begin{tabular}{|c|c|c|c|c|c|}
\hline $\begin{array}{c}\text { Type of } \\
\text { deformation }\end{array}$ & FOA & $\begin{array}{c}\text { Elementary } \\
\text { theory }\end{array}$ & FEM & $\begin{array}{c}\text { \% error to } \\
\text { elementary } \\
\text { theory }\end{array}$ & $\begin{array}{c}\text { \% error to } \\
\text { FEM }\end{array}$ \\
\hline Tension & $1.510 \mathrm{e}-2$ & $1.510 \mathrm{e}-2$ & $1.480 \mathrm{e}-2$ & 0.00 & 2.03 \\
\hline Deflection & 6.518 & 6.471 & 6.270 & 0.73 & 3.96 \\
\hline Twisting angle & $1.340 \mathrm{e}-2$ & $1.384 \mathrm{e}-2$ & $1.199 \mathrm{e}-2$ & 2.90 & 11.76 \\
\hline
\end{tabular}

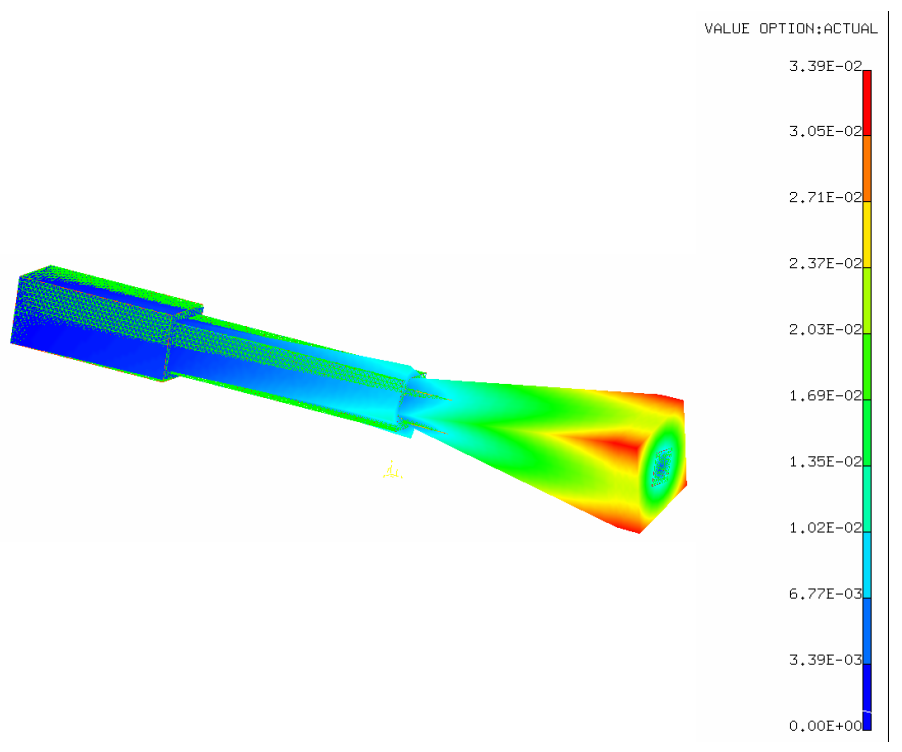

Fig.6 Typical twisting deformation including warping and stress distribution contour of stepped cantilever (enlarged)

hand, the FEM results show some differences because of the stress concentration, the shear deformation and the warping in the twisting deformation as shown in Fig.6. From these results, the FOA element suggested in this paper can estimate properly the simple deformations of tension/compression, bending and twisting approximately. 


\subsection{Tapered Cantilever Beam}

Next let us consider a tapered cantilever structure whose larger portion $n h$ is fixed and applied a tensile force $F$, a shear force $Q$ and a twisting moment $T$ at a tip with smaller cross-section, as shown in Fig.7. When $L / h=4.0, b / h=1.0$ are fixed, the ratio $n$ of height at the support to that at the tip is gradually increased from 1.25 to 2.0. As an example, the height is set as $h=10 \mathrm{~mm}$, and $F=Q=1,000 \mathrm{~N}$ and $T=1,000 \mathrm{Nmm}$ are applied at the smaller end simultaneously. Table 2 shows the numerical results of FOA descritized into one element and that of FEM with more than 108,000 elements for the case when $n=1.25$ and Poisson's ratio is set as 0.3 .

The tip deformation of FOA when $n=1.25,1.5,1.75$ and 2.0, are compared with the finite element results in Fig.8. The larger the taper ratio is, the less the errors of the elongation and the twisting angle are, and the more the error of the deflection is. It is evident from these results that the proposed FOA can estimate the approximate deformation within permissible errors.

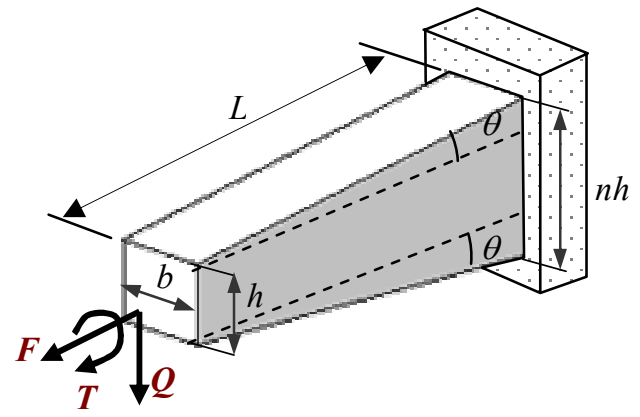

Fig.7 Tapered cantilever beam

Table 2 Deformation analysis of tapered cantilever when $n=1.25$

\begin{tabular}{|c|c|c|c|}
\hline Type of deformation & FOA & FEM & \% error to FEM \\
\hline Tension & $1.785 \mathrm{e}-3$ & $1.7200 \mathrm{e}-3$ & 3.78 \\
\hline Deflection & $8.198 \mathrm{e}-2$ & $8.1800 \mathrm{e}-2$ & 0.22 \\
\hline Twisting angle & $2.9824 \mathrm{e}-4$ & $2.7719 \mathrm{e}-4$ & 7.58 \\
\hline
\end{tabular}

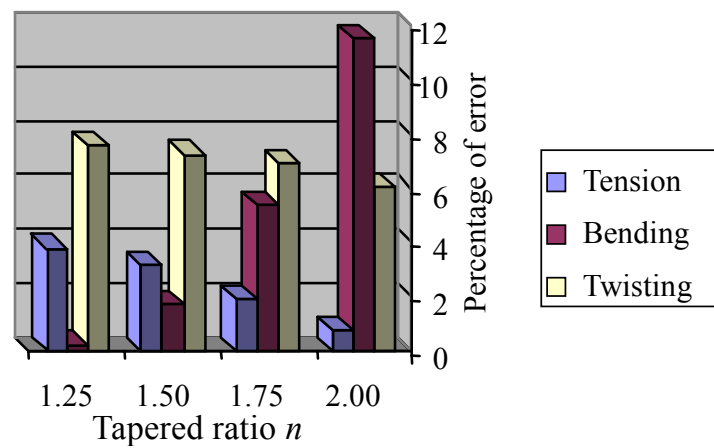

Fig.8 Comparison of differences between FOA and FEM results of tapered cantilever

\subsection{Assembled Structure Applied a Couple of Load Simultaneously}

Next, we have applied the proposed method to more practical and complicate three-dimensional assembled structure, which consists of three taper components and five block components with square cross-section $\left(h^{2}\right)$ as shown in Fig.9, in which the end surfaces $\mathrm{A}$ and $\mathrm{D}$ of the model are fixed, and uniform pressure $2 F / h^{2}$ and line load $Q / h$ are applied on the other end surfaces $\mathrm{B}$ and $\mathrm{C}$ when $F=5 Q$. 


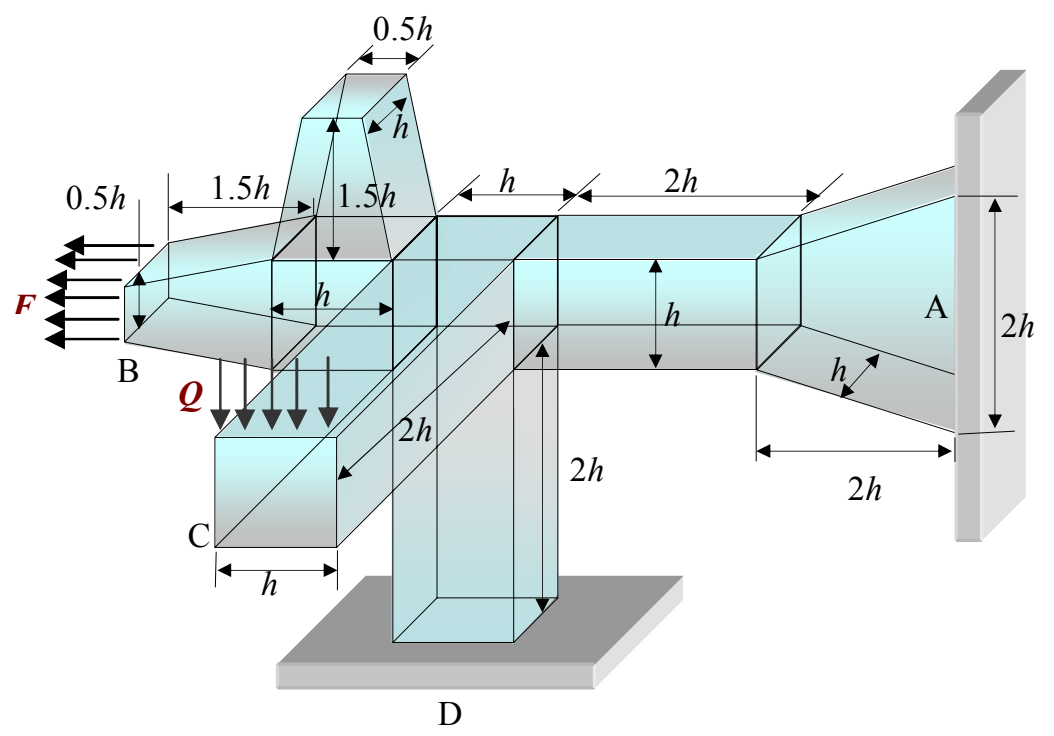

Fig.9 3D-assembled structure model of three tapered blocks and five rectangular blocks with square cross-section

The simulation of this model has been performed by the proposed FOA, when $h=10$ $\mathrm{mm}$ and $F=500 \mathrm{~N}$. This model has been descritized into only eight FOA elements. The results are compared again with the results of usual FEM when $v=0.3$. We have gotten the maximum displacement $2.979 \times 10^{-3} \mathrm{~mm}$ at $\mathrm{B}$ and deflection $7.872 \times 10^{-3} \mathrm{~mm}$ at $\mathrm{C}$ from the proposed FOA method, respectively, whereas the FEM results are $3.436 \times 10^{-3} \mathrm{~mm}$ and $8.47 \times 10^{-3} \mathrm{~mm}$ at the surface center points. The percentages of error at the end $\mathrm{B}$ and $\mathrm{C}$ are $13.30 \%$ and $7.06 \%$, respectively. From these results it is found that the proposed $3 \mathrm{D}$-body FOA can estimate the rough deformation even though with the small number of elements.

\subsection{Simplified Machine Tool Model}

Finally we have applied the proposed 3D-body FOA method to a kind of real machine tool structures like a milling machine as shown in Fig.10(a). The rough sizes of the model are $2.0 \mathrm{~m}$ in height, $1.0 \mathrm{~m}$ in width and $1.5 \mathrm{~m}$ in depth. The model is fixed on the bottom surface and is applied a pair of cutting forces $4.0 \times 10^{3} \mathrm{~N}$ in the opposite directions at the center point A of tool holder and a center point B of work table as shown in the figure. The milling machine model structure has been descritized into 43 3D-body FOA elements as shown in Fig.10(b), and the deformation at the loading points are compared with the results of usual FEM using the mesh of Fig.10(c). The displacements in the force direction at A and $B$ are obtained as $1.75 \mathrm{~mm}$ and $-1.17 \mathrm{~mm}$ by the FOA, and $1.09 \mathrm{~mm}$ and $-1.62 \mathrm{~mm}$ by the FEM, respectively. The percentages of error at $A$ and $B$ are $60.5 \%$ and $27.8 \%$. Figure $10(d)$ shows the axial element forces distribution along the FOA elements, in which the values of axial forces are indicated by the thickness of solid lines. From this figure, it is seen that the bending moment caused by the cutting force at the tool holder is transmitted by a pair of tensile and compressive axial forces along the backside column to the base structure. It is noticed that the proposed 3D-body FOA method can estimate the rough deformation and the loading path with small number of FOA elements.

Even though the FOA formulation of 3D-body has been established, some issues such that how to divide the models into the sub-regions of 3D-blocks, how to place the nodes of FOA elements and how to construct the proposed beam-type FOA elements as well as the precision of the FOA method are still remained. The authors expect to discuss as the future works. 


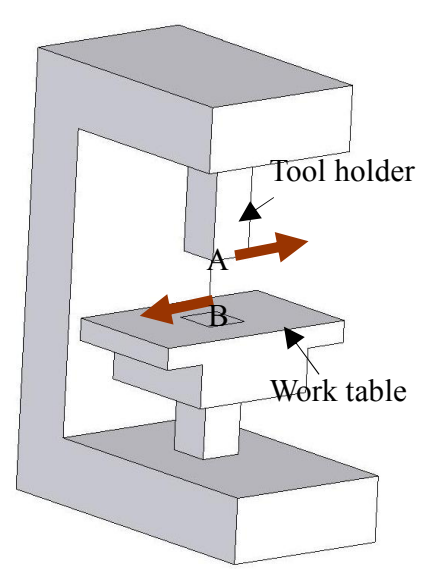

(a) Loading condition

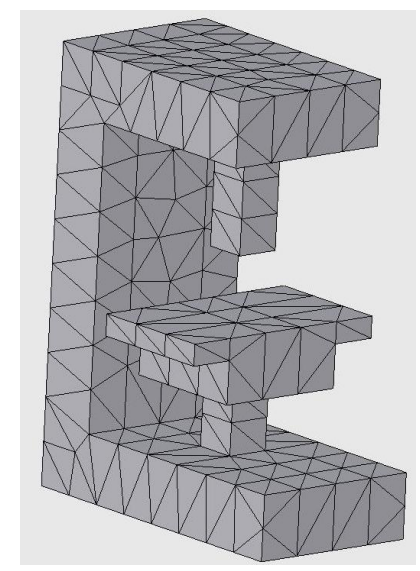

(c) FEM model

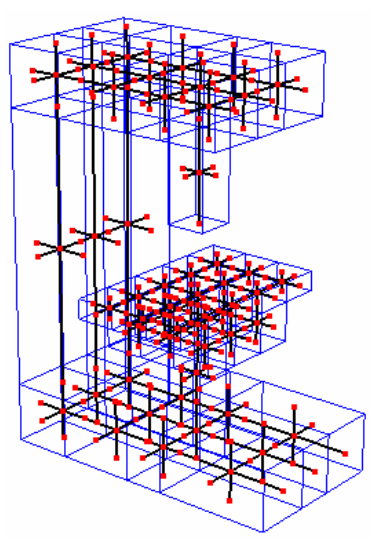

(b) FOA model

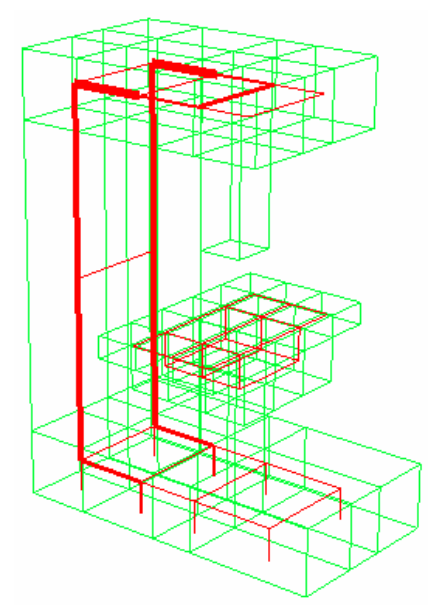

(d) Axial loading paths

Fig.10 Structural model of milling machine

\section{Conclusions}

In this paper, a beam type 3D-body First Order Analysis element as an assembly of six beam elements is proposed to analyze the deformation and the loading path of complicate three-dimensional structures, and the equivalent stiffness of axial, bending and torsional deformation have been formulated. Then the proposed FOA element has been applied to analyze the deformation of several kinds of typical structures. From these numerical results, it is found that the proposed 3D-body FOA method can estimate the approximated global deformation of three-dimensional complicate structures roughly, that are useful for considering the load transmitting path of the structure and for designing a better structure by changing the stiffness of structural components.

\section{References}

(1) Lemon, J.R., Tolani, S.K. and Klosterman, A.L., "Integration and Implementation of Computer Aided Engineering and Related Manufcaturing Capabilities into Mechanical Product Development Process", Gi-Jahrestagung, (1980).

(2) Zienkiewits, O.C., The Finite Element-Method, $3^{\text {rd }}$ ed., (1977), McGraw-Hill, p268-302.

(3) Hughes, T.J.R., The Finite Element Method-Linear Static and Dynamic Finite Element Analysis-, Prientice-Hall, pp.242-262.

(4) Nishigaki, H., Nishiwaki, S., Amago T. and Kikuchi, N., "First Order Analysis for 
Automotive Body Structure Design", Proc. of ASME DETC, DAC 14533(2000), Maryland.

(5) Nishigaki, H., "First Order Analysis for Automotive Body Structure Design Using Excel", $R \& D$ Review of Toyota CRDL, Vol.37, No.1(2001), pp.1-8.

(6) Balabanov, V. and Venter, G., "Multi-Fidelity Optimization with High-Fidelity Analysis and Low-Fidelity Gradients", 10th AIAA/ISSMO Multidisciplinary Analysis and Optimization Conference, AIAA Paper 2004-4459(2004), Albany, NW.

(7) Eldred, M. and Dunlavy, D., "Formulations for Surrogate-Based Optimization with Data Fit, Multifidelity, and Reduced-Order Models", 11th AIAA/ISSMO Multidisciplinary Analysis and Optimization Conference, AIAA Paper 2006-7117 (2006), Portsmouth, VA.

(8) Microsoft Corporation, Microsoft Excel/Visual Basic Reference, 2nd Ed. (1998), Microsoft press.

(9) Bathe, K. J., Finite Element Procedures, (1995), Prentice-Hall, Englewood Cliffs.

(10) Washizu, K., et al. ed., Handbook of finite element method, (1981), Baifukan.(in Japanese) 